\title{
Toluidine blue-containing polymers exhibit potent bactericidal activity when irradiated with red laser light
}

\author{
Stefano Perni, ${ }^{a}$ Polina Prokopovich, ${ }^{b}$ Clara Piccirillo, ${ }^{c}$ Jonathan Pratten, ${ }^{a}$ Ivan P. Parkin ${ }^{* c}$ \\ and Michael Wilson*a
}

\author{
Received 17th November 2008, Accepted 9th February 2009 \\ First published as an Advance Article on the web 9th March 2009 \\ DOI: $10.1039 / b 820561 b$
}

Toluidine blue and toluidine blue-nanogold mixtures were incorporated into polyurethane and silicone polymers by a swell-encapsulation-shrink method using acetone-water mixtures. The surface and mechanical properties of the polymers were changed by the swell-shrink process especially the Young's modulus, but not by the introduction of toluidine blue or nanogold. The antibacterial properties of the various polymers were assessed under laser irradiation at $634 \mathrm{~nm}$ against Escherichia coli and methicillin-resistant Staphylococcus aureus (MRSA). The toluidine blue-incorporated polymers showed kills of $\left(>10^{5} \mathrm{cfu} / \mathrm{ml}\right)$ for MRSA after just one minute of exposure. This is, to our knowledge, the most potent light-activated antimicrobial polymer combination reported to date.

\section{Introduction}

Photosensitisers are a class of chemicals capable of producing free radicals and reactive oxygen species under the influence of light, either from a white light source or from a laser. This property has been widely used in cancer treatment and has also been used in the treatment of oral diseases, ${ }^{1}$ more recently the bactericidal properties of photosensitisers have been successfully investigated in an attempt to combat the rising problem of antibiotic resistance presented by some bacteria. ${ }^{2}$ The multitarget mechanism of interaction between the microorganism and the singlet oxygen/free radicals should minimize the risk of bacterial resistance development to the therapy. Toluidine blue $\mathrm{O}$ (TBO) is frequently used in studies of photodynamic therapy due to its high efficacy and low cost, its chemical structure is presented in Fig. 1.

The surfaces of catheters are subjected to continual adhesion of microorganisms and subsequently the formation of biofilms. This process results in catheter-associated infections ${ }^{3,4}$ that are both a health risk for the patients and also an economic issue for the hospital. ${ }^{5}$ Such infections are difficult to treat because of the reduced susceptibility to antibiotics of the causative organisms resulting from their presence as biofilms. ${ }^{6,7}$ In the USA, a healthcare cost of $\$ 20,000$ per catheter-related infection is reported from hospital costs alone.

When a polymer, such as those used to produce catheters, is immersed in a liquid the amount of fluid that penetrates into the polymer can be expressed in term of the Hildebrand factor. ${ }^{8}$ During the swelling process other molecules dissolved in the

${ }^{a}$ Division of Microbial Diseases, UCL Eastman Dental Institute, University College London, 256 Gray's Inn Road, London, UK WC1X 8LD. E-mail: m.wilson@eastman.ucl.ac.uk

${ }^{b}$ Wolfson School of Mechanical and Manufacturing Engineering, Loughborough University, Loughborough, UK LE11 $3 T U$

${ }^{c}$ Materials Chemistry Research Centre, Department of Chemistry, University College London, 20 Gordon Street, London, UK WC1H OAJ. E-mail: i.p.parkin@ucl.ac.uk<smiles>Cc1cc2c(cc1N)SC1=CC(=[N+](C)C)C=CC1N2</smiles>

Fig. 1 Chemical structure of TBO. ${ }^{24}$

liquid can also penetrate the polymer. When the polymer is removed from the liquid, the solvent evaporates (resulting in shrinking) and the molecules remain encapsulated inside the polymer matrix, provided they are not volatile. This process, called swell-encapsulation-shrink, can be used to incorporate photosensitisers into polymers. The quantity of photosensitiser remaining in the polymer depends on the particular combination of solvent/material/photosensitiser used.

Recently Perni et al. ${ }^{9}$ have shown that methylene blue and methylene blue-nanogold can be embedded in silicone by swelling the polymer in a solution of acetone containing the photosensitiser and that this material is capable of killing bacteria when irradiated with laser light. For example a $3.5 \mathrm{log}$ reduction in MRSA was seen after 8-10 minutes of illumination with a laser source. This study also showed that nanogold had no antimicrobial activity on its own but did synergistically enhance the kill achieved when mixed with methylene blue.

In this paper we report the successful incorporation of a different light-activated antimicrobial (LAA) agent, TBO, together with nanogold in two of the most common materials used to make catheters i.e. silicone and polyurethane. When exposed to laser light, the materials were able to achieve substantial kills of both $E$. coli and MRSA when suspensions of these organisms were placed on the surface of the polymers. The effect of the swelling-shrinking procedure on the Young's modulus, surface energy properties and the friction factor of these polymers was also determined. These new polymer systems are, to our knowledge, the most potent light-activated surfaces 
reported to kill bacteria, with for example complete eradication of MRSA after one minute's exposure to laser light - a ten fold reduction in kill time compared with that previously reported for methylene blue.

\section{Results}

\section{Material characterisation}

Images of the samples before the swelling process and after the shrinking are shown in Fig. 2. It was evident that polyurethane swelled more than silicone and this, amongst other factors, resulted in a higher uptake of TBO. This is illustrated in the UVvisible spectra in Fig. 3 where the absorbance of the polyurethane samples was significantly higher than that of the silicone ones, almost reaching the saturation of the signal. For the silicone sample, the wavelength of highest absorption was $\lambda_{\max }=610 \mathrm{~nm}$, with a shoulder peak at about $510 \mathrm{~nm}$. For the polyurethane sample this wavelength was determined by taking a different UV measurement, where only about one third of the light source area was covered with the sample (thin solid line); in this way the signal was not saturated. This spectrum showed that the wavelength of maximum absorption for polyurethane was $\lambda_{\max }=670$ $\mathrm{nm}$, with a weaker signal at $610 \mathrm{~nm}$. For both materials the UV spectra are different from the one of TBO in an acetone:water solution, where a maximum is detected at $633 \mathrm{~nm}$ (data not shown). This shift is most probably due to the change of the dielectric constant of the surrounding media - aqueous or polymer. With both the silicone and polyurethane polymers, the samples containing TBO and nanogold showed a slightly lower absorbance than that of the sample containing only TBO. The nanogold used had no UV-visible absorption peaks as the particle size of the nanogold at $2 \mathrm{~nm}$ is below the plasmon resonance limit for SPR.

The amount of TBO adsorbed by silicone was approximately $0.003 \mathrm{mg} \mathrm{TBO} / \mathrm{g}$ silicone, whilst polyurethane adsorbed $0.033 \mathrm{mg}$ $\mathrm{TBO} / \mathrm{g}$ polyurethane; this was determined following the procedure described in the experimental section; the distribution of TBO was uniform through the $1 \mathrm{~mm}$ thick silicone and polyurethane samples by optical microscopy when we cut them and looked at the colour side-on.

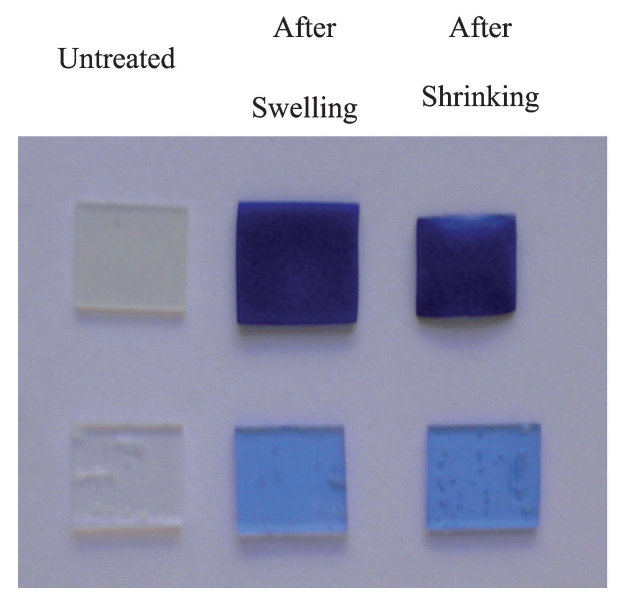

Polyurethane

Fig. 2 Images of untreated polymers and polymers after the swellingshrinking process (untreated samples $1 \times 1 \mathrm{~cm}$ ).
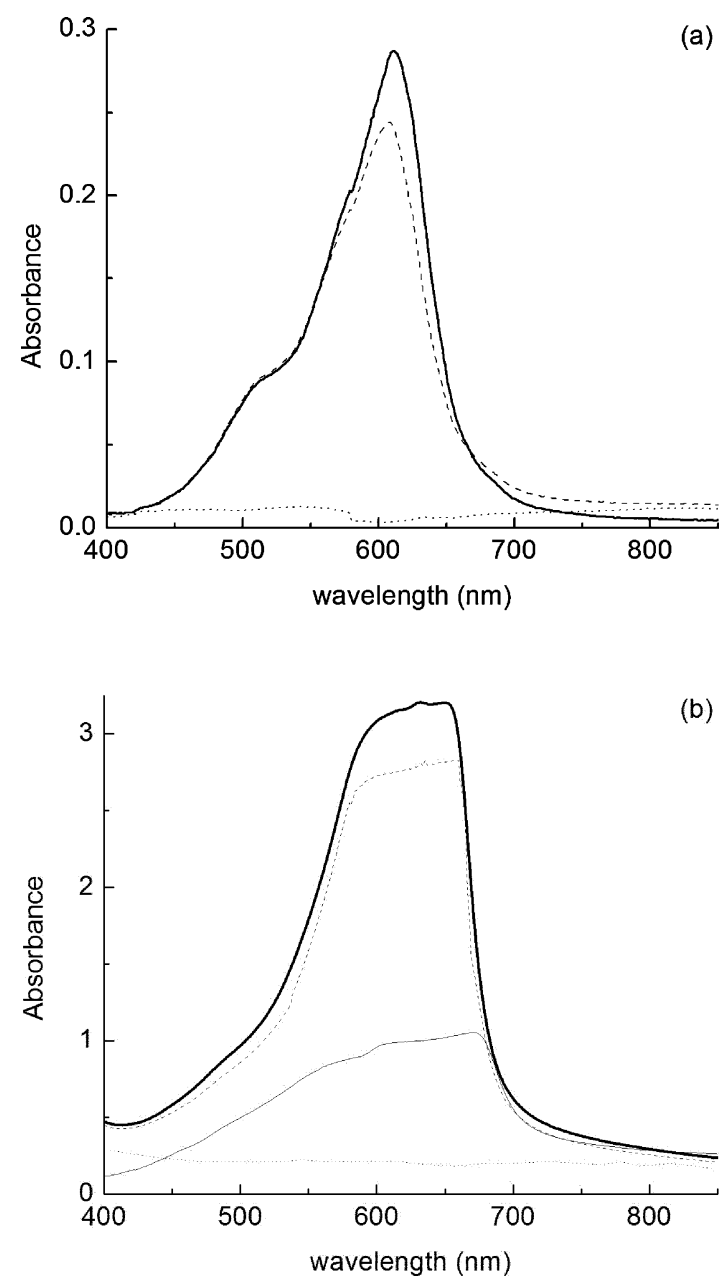

Fig. 3 UV spectra of silicone (a) and polyurethane (b) sample: dotted line: untreated polymer, continuous thick line: polymer with TBO, dashed line: polymer with TBO and Au nanoparticles. For Fig. 3(b) only: continuous thin line: spectrum for polymer with $\mathrm{TBO}$ registered with only one third of the light source covered by the sample.

The contact angles of three liquids (water, hexadecane and ethylene glycol) are shown in Table 1, in general, water presented the highest value of contact angle whilst hexadecane the lowest. The silicone polymer showed a lower contact angle for water droplets after swell-evaporation in an acetone:water mixture. When nanogold was present, the water contact angle of both the silicone and polyurethane polymers was reduced indicating higher hydrophilicity. The presence of TBO made the surface of silicone and polyurethane more hydrophobic (higher contact angle); the presence of both TBO and nanogold resulted in a greater hydrophobicity than that of samples swollen in only acetone:water but not as much as that of samples swollen in only TBO.

Silicone The values of the surface energy parameters are presented in Table 2; the main component is $\gamma^{\mathrm{LW}}$, whilst $\gamma^{\mathrm{AB}}$ was always small. The presence of nanogold increased the value of $\gamma_{\mathrm{S}}{ }^{-}$, the presence of TBO resulted in an increase in the value of $\gamma_{\mathrm{S}}{ }^{+}$. The swelling process was responsible for increasing the surface energy of polyurethane and decreasing that of silicone. 
Table 1 Contact angle $\left(^{\circ}\right) \pm \mathrm{SD}$ of different solvents on the various silicone and polyurethane polymers

\begin{tabular}{lrll}
\hline $\begin{array}{l}\text { Solvent } \\
\text { Silicone }\end{array}$ & \multicolumn{1}{l}{ Water } & Hexadecane & Ethylene glycol \\
\hline Not swollen & $76.5 \pm 0.4$ & $34.2 \pm 0.5$ & $44.7 \pm 0.6$ \\
Swollen acetone:water & $75.3 \pm 0.5$ & $40.5 \pm 0.6$ & $65.1 \pm 0.5$ \\
Swollen acetone:Au & $60.0 \pm 0.4$ & $34.5 \pm 0.4$ & $63.0 \pm 0.8$ \\
Swollen TBO & $104.2 \pm 0.5$ & $35.4 \pm 0.7$ & $82.0 \pm 0.8$ \\
Swollen TBO + Au & $95.0 \pm 0.8$ & $33.0 \pm 0.4$ & $74.8 \pm 0.7$ \\
\hline Polyurethane & & & \\
\hline Not swollen & $92.0 \pm 0.6$ & $38.5 \pm 0.4$ & $77.5 \pm 0.8$ \\
Swollen acetone:water & $86.5 \pm 0.8$ & $28.1 \pm 0.5$ & $64.5 \pm 0.7$ \\
Swollen acetone:Au & $76.0 \pm 0.9$ & $24.0 \pm 0.5$ & $63.6 \pm 0.8$ \\
Swollen TBO & $90.0 \pm 1.0$ & $18.5 \pm 0.4$ & $73.2 \pm 0.9$ \\
Swollen TBO + Au & $87.5 \pm 0.6$ & $17.0 \pm 0.5$ & $68.0 \pm 0.8$ \\
\hline
\end{tabular}

Table 2 Surface energy parameters of silicone and polyurethane polymers

\begin{tabular}{|c|c|c|c|c|c|}
\hline Silicone & $\begin{array}{l}\gamma_{\mathrm{s}}^{\mathrm{LW} /} \\
\mathrm{mJ} \mathrm{m}^{-2}\end{array}$ & $\begin{array}{l}\gamma_{\mathrm{s}}^{+} / \\
\mathrm{mJ} \mathrm{m}^{-2}\end{array}$ & $\begin{array}{l}\gamma_{\mathrm{s}^{-}} / \\
\mathrm{mJ} \mathrm{m}^{-2}\end{array}$ & $\begin{array}{l}\gamma_{\mathrm{S}}^{\mathrm{AB} /} \\
\mathrm{mJ} \mathrm{m}^{-2}\end{array}$ & $\begin{array}{l}\gamma_{\mathrm{S}}^{\mathrm{TOT} /} \\
\mathrm{mJ}^{-2}\end{array}$ \\
\hline Untreated & 22.70 & 2.79 & 8.12 & 9.51 & 32.22 \\
\hline Swollen acetone:water & 21.18 & 0.24 & 18.71 & 4.24 & 25.42 \\
\hline Swollen acetone:Au & 22.65 & 0.01 & 39.96 & 0.89 & 23.54 \\
\hline ТВO & 22.37 & 0.01 & 0.96 & 0.15 & 22.52 \\
\hline $\mathrm{TBO}+\mathrm{Au}$ & 22.99 & 0.09 & 3.36 & 1.10 & 24.09 \\
\hline \multicolumn{6}{|l|}{ Polyurethane } \\
\hline Untreated & 21.64 & 0.01 & 6.58 & 0.48 & 22.13 \\
\hline Swollen acetone:water & 24.09 & 0.43 & 6.04 & 3.22 & 27.31 \\
\hline Swollen acetone: $\mathrm{Au}$ & 24.99 & 0.11 & 16.11 & 2.66 & 27.65 \\
\hline TBO & 25.81 & $<0.01$ & 6.24 & 0.09 & 25.89 \\
\hline $\mathrm{TBO}+\mathrm{Au}$ & 26.03 & 0.09 & 6.31 & 1.49 & 27.53 \\
\hline
\end{tabular}

The AFM images of the surface of the samples are in Fig. 4; The surface roughness (Table 3 ) of both the silicone and polyurethane polymers increased about $25 \%$ after swelling in acetone:water, this difference is significant $(p>0.05)$; no differences were recorded when the swelling solution contained TBO and/or nanogold $(p>0.05)$.

The friction coefficient (Table 3) of silicone was lower than that of polyurethane. The swelling process did not significantly alter the friction coefficient as the value of the untreated material was not significantly different from any of the swollen materials $(p>0.05)$. The Young's modulus of silicone nearly doubled after swelling in acetone:water, however the presence of either nanogold or TBO in the solution did not cause any additional effect on the value of the Young's modulus. In contrast, the value of Young's modulus for polyurethane was reduced to almost one half that of the untreated samples. For both materials there were not significant differences among the values of Young's modulus of samples swollen in any of the solutions employed $(p>0.05)$, furthermore these values were significantly different from the untreated material.

The singlet oxygen assay (Table 4) revealed that only the combination of TBO and laser light were responsible for the generation of singlet oxygen, as the untreated samples and those swollen in only acetone:water or acetone:Au did not result in the formation of singlet oxygen. The amount of singlet oxygen generated was greater in the case of polyurethane than silicone $(p>0.05)$ and for both polymers the presence of nanogold increased slightly the singlet oxygen produced when irradiated but this difference was not statistically significant $(p>0.05)$. The amount of singlet oxygen produced did not scale linearly with the concentration of the TBO in the polymer, but in general a higher amount of TBO led to a higher measurement.

\section{Bactericidal assays}

The viable cell counts of Escherichia coli and methicillin-resistant Staphylococcus aureus (MRSA) deposited on the surfaces of the silicone and polyurethane polymers are presented in Fig. 5 and Fig. 6. It is evident that the number of viable cells recovered after exposure to laser light decreased with exposure time when TBO was present in the polymeric matrix $(\mathrm{L}+\mathrm{TBO}+)$. The reduction in the viable count of $E$. coli was greater on polyurethane than on silicone and amounted to $>4 \log _{10}$ after 2 min exposure and $1.5 \log _{10}$ after $3 \mathrm{~min}$ exposure respectively. Similarly, for MRSA the detection limit (reduction $>4 \log _{10}$ ) was reached after 3 min when silicone was employed and after $1 \mathrm{~min}$ when polyurethane was used. No reduction in the viable count was recorded with either silicone or polyurethane when TBO was absent (L+TBOand $\mathrm{L}-\mathrm{TBO}-$ ) or when TBO was not present and the polymer was not exposed to laser light (L-TBO-). The killing of E. coli and MRSA was not significantly enhanced by the presence of nanogold in either silicone or polyurethane.

\section{Discussion}

Biofilm formation on catheters and other medical devices is regarded as a major cause of hospital-acquired infections. ${ }^{3,4}$ Many approaches have been used in order to produce materials that are able to reduce or prevent biofilm formation on such devices and include the incorporation of silver ions ${ }^{10}$ or through the release of an antimicrobial compound from the surface. ${ }^{11}$ These methods are effective but their mode of action is ultimately likely to result in the development of resistance in the target organisms. ${ }^{12}$ Lethal photosensitisation of microbes, however, generates reactive oxygen species that damage a range of microbial components and processes ${ }^{13}$ and thereby reduces the opportunity for the organism to develop resistance against this approach. The incorporation of a photosensitiser such as TBO into a polymer, therefore, could result in an antimicrobial material that would be useful for the fabrication of catheters and other medical devices requiring protection from biofilm formation. In this study we examined the properties of two photosensitiser-containing polymers using materials frequently used in the fabrication of catheters - silicone and polyurethane.

The higher uptake of TBO shown by polyurethane is in part due to the increased swelling that this material achieves compared to that of silicone; other factors (such as the dimensions and the shapes of the pores) can also have an effect. The swelling depends on the affinity of the solution towards the material and the amount of solvent penetrating into the polymer matrix. This correlates directly with the TBO uptake. Other 

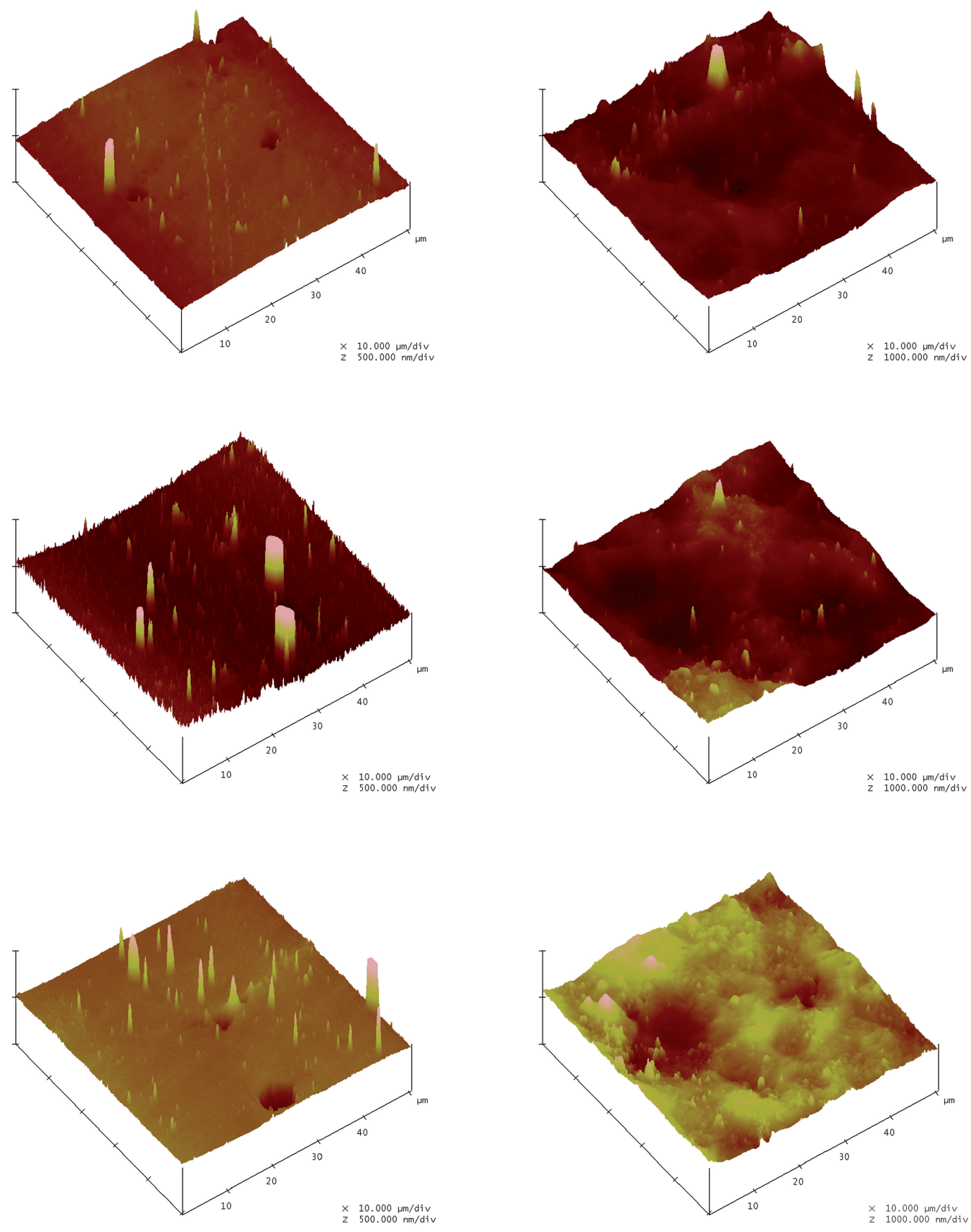

Fig. 4 AFM images of untreated silicone (a) untreated polyurethane (b) silicone with TBO (c) polyurethane with TBO (d) silicone with TBO and nanogold (e) polyurethane with TBO and nanogold (f).

solvents were tested but did not result in significant uptake of TBO. Both methanol and water, for example, did not achieve a significant uptake of TBO despite the solubility of TBO being higher in these solvents than acetone and these allow higher initial TBO concentrations to be used.

We showed that, in general, the physical properties of the materials were only slightly affected by the process of swelling in an organic solvent solution containing the photosensitiser. Moreover, we found that the swelling alone was responsible for these changes rather than the presence of TBO or nanogold, similar to the effect of Methylene Blue when embedded in silicone, as previously reported. ${ }^{9}$ This was the case with respect to both the surface roughness and elastic modulus. The intense swelling of polyurethane is probably responsible for the reduction of the Young's modulus in the treated samples.

Insertion of catheters into a patient generates frictional forces and these are an important factor in determining patient comfort, ${ }^{14,15}$ the correlation between applied force and friction force is described by their ratio known as the friction coefficient; AFM has been employed to determine the friction coefficients of surfaces and to assess if a specific treatment affects it. ${ }^{16} \mathrm{We}$ demonstrated here that the swelling-shrinking process does not 
Table 3 Surface roughness, friction coefficients (applied force $=15.0$ $\mathrm{nN}$ ) and Young's modulus of silicone and polyurethane polymers. Values show average $\pm \mathrm{SD}$

\begin{tabular}{llll}
\hline & $R a / \mu \mathrm{m}$ & $\begin{array}{l}\text { Friction } \\
\text { coefficient }\end{array}$ & $\begin{array}{l}\text { Young's } \\
\text { module/MPa }\end{array}$ \\
Silicone & & & \\
\hline Untreated & $0.031 \pm 0.007$ & $0.21 \pm 0.02$ & $0.56 \pm 0.04$ \\
Swollen acetone:water & $0.052 \pm 0.006$ & $0.23 \pm 0.03$ & $1.12 \pm 0.08$ \\
Swollen acetone:Au & $0.045 \pm 0.006$ & $0.26 \pm 0.02$ & $1.02 \pm 0.06$ \\
TBO & $0.040 \pm 0.005$ & $0.25 \pm 0.02$ & $0.99 \pm 0.07$ \\
TBO + Au & $0.042 \pm 0.008$ & $0.25 \pm 0.01$ & $1.01 \pm 0.05$ \\
\hline Polyurethane & & & \\
\hline Untreated & $0.191 \pm 0.018$ & $0.28 \pm 0.02$ & $8.77 \pm 0.33$ \\
Swollen acetone:water & $0.249 \pm 0.023$ & $0.32 \pm 0.03$ & $5.24 \pm 0.35$ \\
Swollen acetone:Au & $0.221 \pm 0.034$ & $0.39 \pm 0.04$ & $4.69 \pm 0.29$ \\
TBO & $0.233 \pm 0.029$ & $0.30 \pm 0.02$ & $4.02 \pm 0.27$ \\
TBO + Au & $0.215 \pm 0.037$ & $0.35 \pm 0.01$ & $4.35 \pm 0.20$ \\
\hline
\end{tabular}

Table 4 Singlet oxygen assay and TBO release by the various polymers (variation of absorbance at $293 \mathrm{~nm}$ and $633 \mathrm{~nm}$ ) after 1 minute laser exposure. Values show average \pm SD

\begin{tabular}{lcc}
\hline Silicone & $\Delta A_{293}$ & $\Delta A_{633}$ \\
\hline Untreated & 0.00 & 0.00 \\
Swollen acetone:water & 0.00 & 0.00 \\
Swollen acetone:Au & 0.00 & 0.00 \\
TBO & $-0.11 \pm 0.03$ & 0.00 \\
TBO $+\mathrm{Au}$ & $-0.10 \pm 0.02$ & 0.00 \\
\hline Polyurethane & & \\
\hline Untreated & 0.00 & 0.00 \\
Swollen acetone:water & 0.00 & 0.00 \\
Swollen acetone:Au & 0.00 & 0.00 \\
TBO & $-0.33 \pm 0.05$ & 0.00 \\
TBO + Au & $-0.26 \pm 0.05$ & 0.00 \\
\hline
\end{tabular}

affect the friction coefficient of silicone or polyurethane guaranteeing that catheters made with these materials would not require additional care for insertion.

Photosensitisers produce the greatest yield of reactive oxygen species when irradiated at the specific wavelength corresponding to their absorbance maximum. In this work we used a light source emitting at the absorbance maximum of TBO in water solution i.e. $633 \mathrm{~nm}$. We showed that there was a shift in absorbtion maxima to higher energy when embedded in silicone or polyurethane. The wavelength of the laser light used in this study still had good overlap with TBO when in silicone or polyurethane. This information can be implemented in tuning the laser wavelength to improve the absorbance of the materials and, consequently, the photosensitiser activity.

Knowledge of the surface properties of the resulting materials is important as these can affect bacterial adhesion to surfaces and furthermore they could be usefully employed in preventing biofilm formation ${ }^{17}$ or to choose a material less prone to microbial adhesion. The presence of nanogold tends to increase the hydrophilicity and the surface energy related to the electron donor properties of silicone and polyurethane. Silicone and polyurethane containing TBO were more hydrophilic than the untreated materials, whilst the simultaneous presence of TBO and nanogold resulted in a greater hydrophobicity than the untreated material but this shift was mitigated by the hydrophilic characteristic resulting from the presence of the nanogold.

The effect of TBO in combination with laser light was studied with the appropriate set of control ${ }^{18}$ experiments in which TBOfree polymer was used with light ( $\mathrm{L}+\mathrm{TBO}-$ ), the photosensitiser based polymer without laser light $(\mathrm{L}-\mathrm{TBO}+)$ and when neither laser light nor photosensitiser were present ( $\mathrm{L}-\mathrm{TBO}-$ ). None of the controls achieved significant killing of the two target organisms. The killing of E. coli and MRSA was significantly greater on the TBO-containing polyurethane polymer than on the TBO-containing silicone, this is attributable to the higher TBO concentration present in the former - furthermore it was this polymer that also produced the greatest yield of singlet oxygen when exposed to light.

Gram-negative bacteria are, in general, more resistant to lethal photosensitisation than Gram-positive species due to their different cell wall structure. ${ }^{19}$ This explains the different levels of killing exhibited against E. coli, a Gram-negative species, compared to MRSA, a Gram-positive species. Such differences in susceptibility to light-activated antimicrobial agents have been reported for E. coli and S. aureus when deposited on photosensitiser-containing cellulose and irradiated with white light. ${ }^{20}$

Previous studies have reported that nanogold can enhance the antimicrobial properties of Methylene Blue when they are both embedded in silicone. ${ }^{9}$ Here we did not notice this effect for TBO in either silicone or polyurethane - this may be attributable to differences in the wavelength of the light used to activate the Methylene Blue $(665 \mathrm{~nm})$ and TBO $(633 \mathrm{~nm})$ which may influence interaction between the photosensitiser and the nanogold, or it could also be related to an underlying difference in mechanism.

The TBO polyurethane polymer reported here exhibited exceptional killing of both $E$. coli and MRSA at very short exposure times to laser irradiation. The kills achieved exceed in log terms everything that has been reported previously for LAApolymer combinations. Furthermore, a complete kill was noted with even very short irradiation times of just one minute in the case of MRSA, this is a ten-fold reduction in kill time compared to our previous work using a silicone polymer and a Methylene Blue-nanogold combination (3.5 log reduction following $10 \mathrm{~min}$ exposure to laser light). Literature precedents for other antimicrobial surfaces in terms of time taken for complete disinfection of a surface are hard to correlate because of different methods of analysis, testing and organism type. However, in our hands hard photoactive coatings such as titanium dioxide and doped titanium dioxide require much longer time periods for significant kill of bacteria such as $S$. aureus of the order of 4-24 hours. Similarly, antimicrobial silver- or copper-doped surfaces typically require hours to achieve the level of disinfection noted in this study.

The time required to prepare these materials could be reduced through performing the swelling and drying at a higher temperature than the $20{ }^{\circ} \mathrm{C}$ currently used. Furthermore, the drying stage could also be carried out under vacuum conditions to increase the flow of solvent from the elastomers. In this way, the speed of the proposed process could be increased and it may be more applicable for scale-up to an industrial level. 
(a)

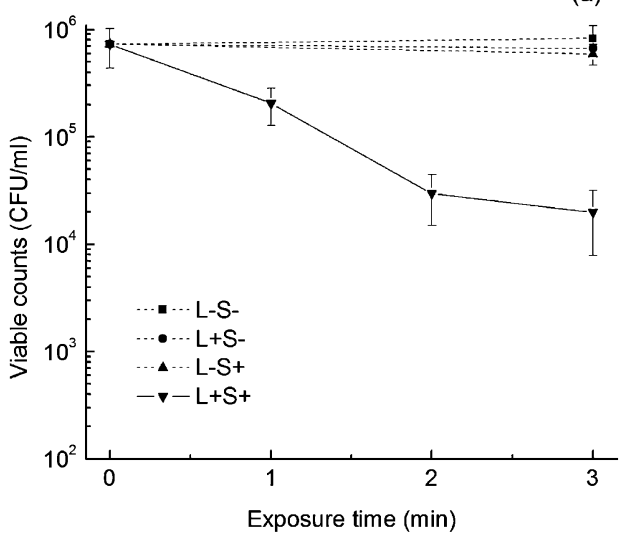

(c)

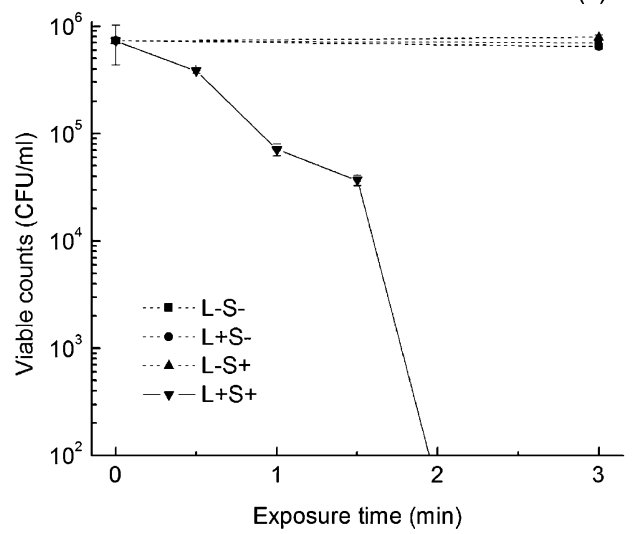

(b)

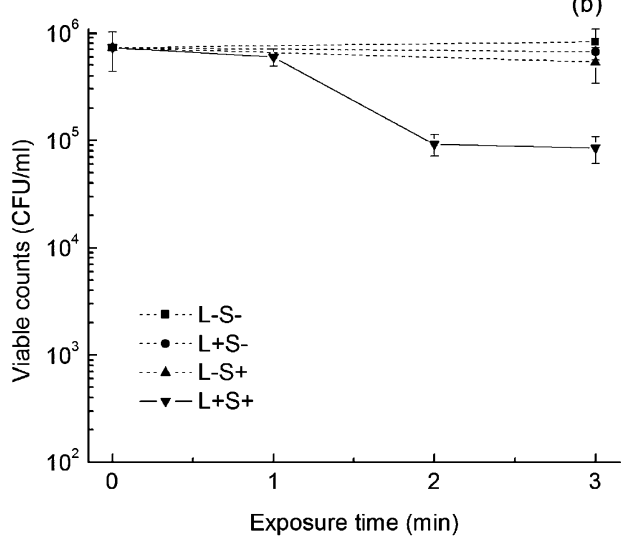

(d)

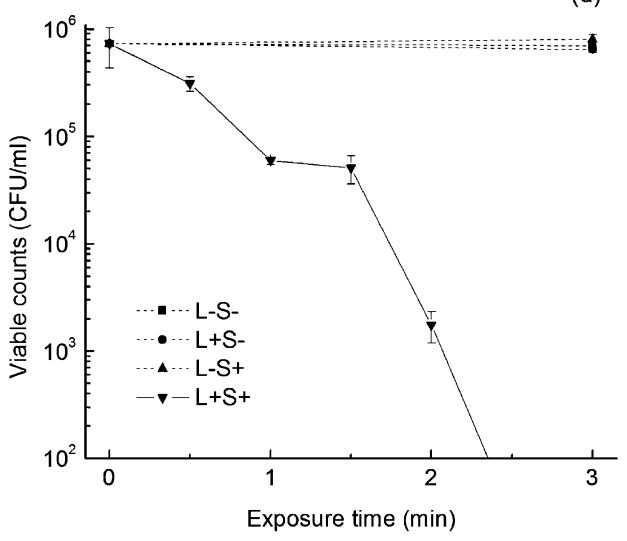

Fig. 5 Concentration of viable Escherichia coli $( \pm \mathrm{SD})$ present on the surfaces of the polymers as a function of the laser exposure time $(\mathrm{L}+=$ laser present, $\mathrm{L}-=$ no laser) or, in the case of controls, incubation for the same period of time in the dark ( $+=$ with TBO; $\mathrm{S}-=$ bare polymer, no TBO). (a) $\mathrm{TBO}$ in silicone; (b) TBO + Au in silicone; (c) TBO in polyurethane; (d) TBO + Au in polyurethane.

\section{Experimental}

\section{Elastomer preparation}

Silicone elastomers were prepared using liquid MED-4850 (Polymer Systems Technology Ltd.) as a starting material; this was mixed with the crosslinking agent in a 1:1 ratio, and spread uniformly on to a glass surface to make a $1 \mathrm{~mm}$ thick sheet. The polymer was then cured at $80^{\circ} \mathrm{C}$ for 2.5 hours. After cooling, the sheet was cut into smaller coupons $(1.0 \times 1.0 \mathrm{~cm}$ squares $)$. Polyurethane samples were cut in squares $1.0 \times 1.0 \mathrm{~cm}$ from a sheet (thickness $0.8 \mathrm{~mm}$ ) purchased from American Polyfilm Inc. (Branford, CT, USA). Both these polymers are the medical grade of silicone and polyurethane respectively and are the two most widely used polymers used to form catheters.

Samples of different dimensions (thinner layer or larger squares) were prepared, if required, for particular characterization analysis.

\section{Preparation of polymers with embedded toluidine blue $\mathrm{O}$ and nanogold}

Toluidine blue O (Sigma, UK) solutions were prepared at a concentration of 500 ppm in acetone: $\mathrm{dH}_{2} \mathrm{O}$ or in acetone:nanoparticles, in both cases the ratio was 9:1 acetone:aqueous solution. The solutions were sonicated in an ultrasound bath for 15 minutes to ensure complete TBO dissolution. $\mathrm{Au}$ nanoparticles were purchased from BBInternational Ltd (Cardiff, UK) and were stated to be $2 \mathrm{~nm}$ in diameter with a particle concentration of $2 \times 10^{15}$ particles per $\mathrm{ml}$.

Several polymer samples were prepared, containing TBO only $(\mathrm{TBO}+\mathrm{Au}-), \mathrm{TBO}$ and $\mathrm{Au}$ nanoparticles $(\mathrm{TBO}+\mathrm{Au}+)$ or nanoparticles only (TBO-Au+). In all cases, a $1.0 \mathrm{~cm}$ square sample was placed into the appropriate solution and left to swell in the dark for 24 hours inside a closed bottle containing $10.0 \mathrm{ml}$ of TBO solution. After this, the samples were left to dry in the dark at room temperature for 24 hours. As blanks, polymer samples not swollen in any solvent and one swollen in the solvents only and allowed to evaporate were also prepared.

\section{Determination of TBO uptake and UV spectra}

UV analysis of both the swelling solutions and the elastomer samples were performed using a UNICAM UV 500 UV/Visible spectrophotometer (ThermoSpectronic, Rochester, NY, USA). The amount of TBO that was absorbed by the two polymers was 
(a)

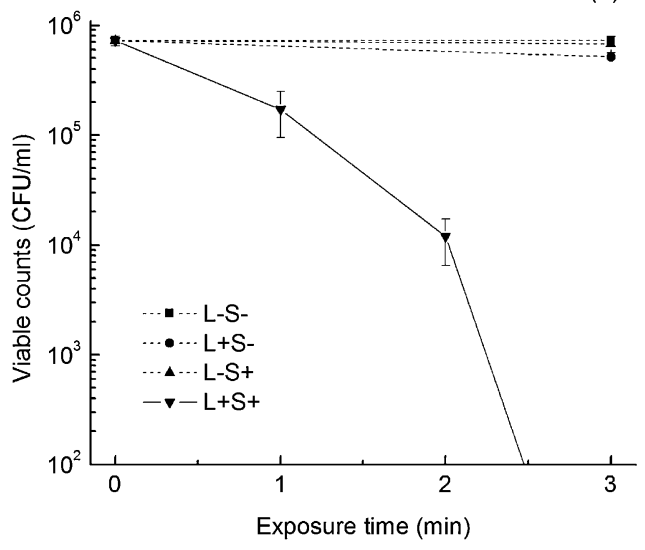

(c)

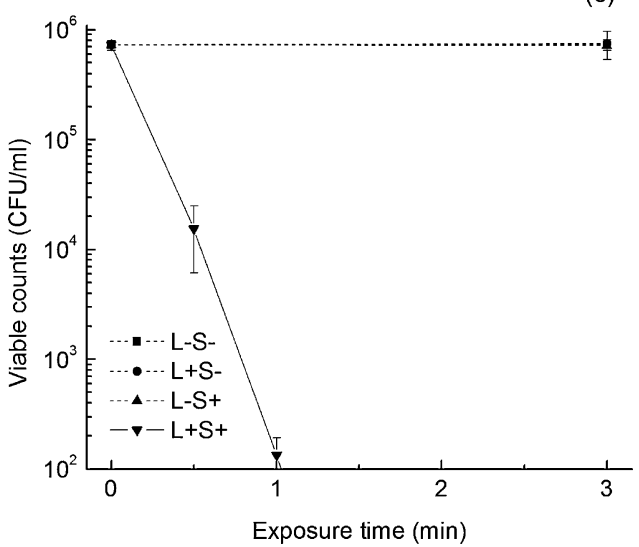

(b)

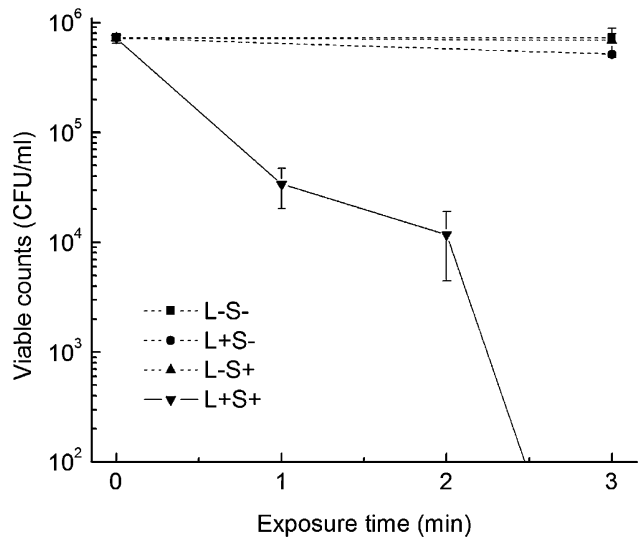

(d)

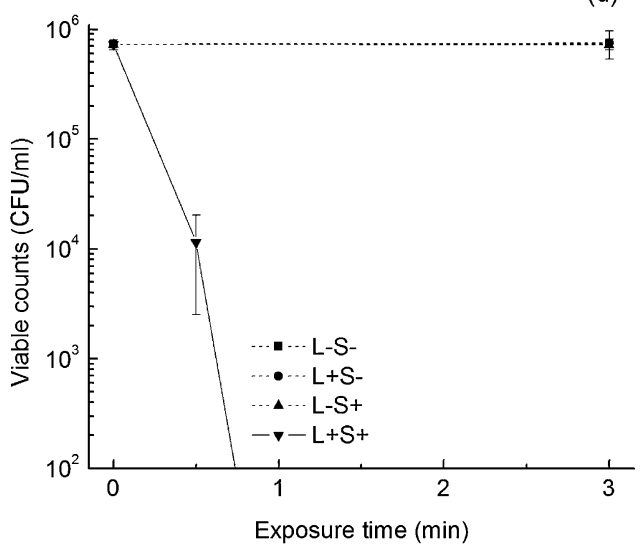

Fig. 6 Concentration of viable methicillin-resistant Staphylococcus aureus $( \pm \mathrm{SD})$ present on the surfaces of the polymers as a function of the laser exposure time $(\mathrm{L}+=$ laser present, $\mathrm{L}-=$ no laser) or, in the case of controls, incubation for the same period of time in the dark ( $\mathrm{S}+=$ with $\mathrm{TBO} ; \mathrm{S}-=$ bare polymer, no TBO). (a) TBO in silicone; (b) TBO + Au in silicone; (c) TBO in polyurethane; (d) TBO + Au in polyurethane.

determined through mass balance; the concentration of TBO in the solution was measured before and after the polymer swelling by UV at $633 \mathrm{~nm}$ against a calibration curve. The amount of TBO absorbed was divided by the weight of the polymer, giving a concentration as $g$ of TBO per $g$ of polymer.

\section{Determination of surface free energy parameters and contact angle measurements}

The total surface free energy $\gamma^{\text {TOT }}$ consists of two components and it is defined as:

$$
\gamma^{\mathrm{TOT}}=\gamma^{\mathrm{LW}}+\gamma^{\mathrm{AB}}
$$

Where $\gamma^{\mathrm{LW}}$ is the apolar component of the surface free energy associated with Lifshitz-Van der Waals interactions and $\gamma^{\mathrm{AB}}$ is the acid-base component of surface free energy. $\gamma^{\mathrm{AB}}$ results from the electron-donor $\left(\gamma^{-}\right)$and electron-acceptor $\left(\gamma^{+}\right)$molecular interactions (i.e. Lewis acid-base interactions). The acid-base term is expressed as the product of the electron donor and electron acceptor parameters:

$$
\gamma^{\mathrm{AB}}=2 \sqrt{\gamma^{+} \gamma^{-}}
$$

The interfacial energy, $\gamma_{\mathrm{SL}}$, is defined as: ${ }^{21}$

$$
\begin{aligned}
\gamma_{\mathrm{SL}}= & \left(\sqrt{\gamma_{\mathrm{S}}^{\mathrm{LW}}}-\sqrt{\gamma_{\mathrm{L}}^{\mathrm{LW}}}\right)^{2}+2\left(\sqrt{\gamma_{\mathrm{S}}^{+} \gamma_{\mathrm{S}}^{-}}\right. \\
& \left.+\sqrt{\gamma_{\mathrm{L}}^{+} \gamma_{\mathrm{L}}^{-}}-\sqrt{\gamma_{\mathrm{S}}^{+} \gamma_{\mathrm{L}}^{-}}-\sqrt{\gamma_{\mathrm{S}}^{-} \gamma_{\mathrm{L}}^{+}}\right)
\end{aligned}
$$

where the subscripts $\mathrm{S}$ and $\mathrm{L}$ refer to the solid and liquid phases, respectively. The Young equation can be combined with the Young-Dupre equation to yield eqn (4).

$$
\gamma_{\mathrm{L}}(1+\cos \theta)=\gamma_{\mathrm{S}}+\gamma_{\mathrm{L}}-\gamma_{\mathrm{SL}}=-\Delta G_{\mathrm{SL}}
$$

Substituting the appropriate expressions then gives eqn (5):

$$
\gamma_{\mathrm{L}}(1+\cos \theta)=2\left(\sqrt{\gamma_{\mathrm{S}}^{\mathrm{LW}} \gamma_{\mathrm{L}}^{\mathrm{LW}}}+\sqrt{\gamma_{\mathrm{S}}^{+} \gamma_{\mathrm{L}}^{-}}+\sqrt{\gamma_{\mathrm{S}}^{-} \gamma_{\mathrm{L}}^{+}}\right)
$$

The equilibrium spreading pressure $\left(\pi_{\mathrm{e}}\right)$ was assumed to be negligible. A set of three simultaneous equations can then be solved to obtain the surface energy parameters of the solid, by using the known parameters of the three liquids and their contact angles on a solid. ${ }^{22}$

Equilibrium contact angle measurements of distilled water, ethylene glycol, hexadecane (Sigma, UK) were performed with 
a G40 goniometer (Kruss, Town, France) at a temperature of 23 $+0.5^{\circ} \mathrm{C}$ and a relative humidity of $40 \%$. For each material, ten replicates on fresh samples were analysed.

\section{AFM measurements of surface roughness and friction coefficient}

Atomic Force Microscopy (Nanoscope IIIa, Digital Instruments) was used to characterize the surface topography (roughness) and to determine the coefficient of friction. All experiments were performed at ambient conditions ( $40 \pm 1 \% \mathrm{RH}$, temperature $20 \pm 0.5^{\circ} \mathrm{C}$ ). Roughness measurements were carried out in tapping mode, while friction measurements were conducted in lateral force mode. V-shaped microfabricated $(100 \mu \mathrm{m})$ cantilevers with pyramidal, oxide-sharpened $\mathrm{Si}_{3} \mathrm{~N}_{4}$ tips, supplied by Digital Instruments (model DNP, spring constant of $0.58 \mathrm{~N} \mathrm{~m}^{-1}$ ) were used for all friction measurements. Surface friction force data was acquired by simultaneously scanning in the forward $(+x)$ and reverse $(-x)$ direction with disabled scanning in the $y$ direction. The scan velocity was $50 \mu \mathrm{m} \mathrm{s}^{-1}$ at a scan frequency equal to $1.0 \mathrm{~Hz}$. Each measurement reported represents an average of at least five independent scans. In order to convert raw friction signals $(\mathrm{V})$ into lateral forces $(\mathrm{N})$, a lateral force calibration factor $\alpha(\mathrm{N} / \mathrm{V})$ was obtained according to the calibration procedure described in literature ${ }^{16}$ using silicone wafers as a calibration standard. The silicone wafers were cleaned for $10 \mathrm{~min}$ in acetone, rinsed with deionised water and dried by adding a few drops of ethanol to remove excess water. The calibration factor was estimated to be $\alpha=0.019 \mathrm{nN} / \mathrm{V}$.

\section{Mechanical properties of the samples}

The mechanical properties (Young modulus) of the samples were studied by performing stress-strain analysis with an Instron 3366 (10 kN capacity) using the mechanical wedge action grips. Samples with section $1 \times 6 \mathrm{~mm}$ thick were stretched at a speed of $350 \mathrm{~mm} / \mathrm{min}$ from an initial distance between the grips of $50 \mathrm{~mm}$; the load applied was measured up to the breaking of the sample.

\section{Singlet oxygen assay}

The protocol used to quantify the singlet oxygen yield produced by each of the elastomer samples was adapted from Fischer et al. ${ }^{23}$ The assay measures the absorbance of uric acid at $293 \mathrm{~nm}$ before and after irradiation; any decrease in the absorbance is directly proportional to the singlet oxygen produced. A fresh stock solution of uric acid (Sigma, Poole, UK) was made up in phosphate buffered saline (PBS) at a concentration of $100 \mu \mathrm{g}$ $\mathrm{mL}^{-1}$ one hour prior to the assay. This stock solution was repeatedly vortexed to ensure mixing and dissolution and was diluted down to a final concentration of $20 \mu \mathrm{g} \mathrm{mL} \mathrm{m}^{-1}$ in PBS. Duplicate samples of each type of polymers $(\mathrm{TBO}+\mathrm{Au}+$, $\mathrm{TBO}+\mathrm{Au}-, \mathrm{TBO}-\mathrm{Au}+$, and $\mathrm{TBO}-\mathrm{Au}-$ ) were placed into individual wells of a 6 well tissue culture plate (Sarstedt, Leicester, UK) and immersed in $5 \mathrm{~mL}$ of the uric acid solution. The samples were thoroughly mixed and then duplicate $1 \mathrm{~mL}$ aliquots were transferred to special UV range cuvettes (JenconsPLS, East Grinstead, UK). The absorbance at $293 \mathrm{~nm}\left(A_{293}\right)$ of each sample was measured using an Ultrospec 2000 UV/Visible spectrophotometer (Amersham Pharmacia Biotech, Piscataway, NJ, USA). Following irradiation of the samples with the laser light for 1 minute, duplicate $1 \mathrm{~mL}$ aliquots were again removed from each sample and the $A_{293}$ was again measured. These measurements were then expressed as the average change in $A_{293}$. In addition, in order to assay leaching of the TBO from the polymer, the absorption of the samples at $633 \mathrm{~nm}$ (the absorption maximum for TBO in PBS) was also measured.

\section{Bactericidal assays}

The organisms used were methicillin-resistant Staphylococcus aureus EMRSA-16 and Escherichia coli ATCC 25922. These organisms were maintained by weekly subculture on Brain Heart Infusion (BHI) agar (Oxoid, Basingstoke, UK). For experimental purposes, the bacteria were grown aerobically in BHI broth (Oxoid) at $37{ }^{\circ} \mathrm{C}$ for 24 hours. These overnight cultures were then diluted 1 in 1000 in PBS. The resulting bacterial suspensions contained approximately $10^{6} \mathrm{cfu} \mathrm{mL}^{-1}$.

The antimicrobial activity of the different elastomer samples was then assessed using the following assay. Triplicate samples of each type of elastomer $(\mathrm{TBO}+\mathrm{Au}+, \mathrm{TBO}+\mathrm{Au}-, \mathrm{TBO}-\mathrm{Au}+$, $\mathrm{TBO}-\mathrm{Au}-$ ) were placed onto raised platforms (plastic lids) inside separate wells of two 6-well tissue culture plates (Sarstedt, Leicester, UK). Aliquots $(25 \mu \mathrm{L})$ of the microbial suspension were carefully placed on the surface of each elastomer sample and a glass cover slip was gently placed on top. After irradiation with light (634 nm) from a $1.0 \mathrm{~W}$ laser (BFi Optilas Ltd., UK) for up to 3 minutes, or incubation for the same length of time in the dark in the case of controls, $10 \mu$ l aliquots of the suspension were plated out onto either Mannitol Salt agar (MSA) in the case of $S$. aureus or MacConkey agar (MAC) in the case of E. coli. The remaining $15 \mu \mathrm{l}$ of suspension was removed from each polymer sample by pipetting and added to $135 \mu \mathrm{l}$ of sterile PBS in a $50 \mathrm{ml}$ tube (Sarstedt). The elastomer samples themselves were then carefully removed and added to the corresponding tubes. All samples were briefly vortexed and further serial dilutions were prepared in sterile PBS. Duplicate $25 \mu$ aliquots of each dilution were then plated out onto either MSA or MAC. All plates were incubated aerobically at $37{ }^{\circ} \mathrm{C}$ for 24 hours and survivors were then enumerated by viable counting.

\section{Statistical analyses}

The Mann-Whitney $U$ test was used to compare the number of survivors recovered from the various silicone and polyurethane samples $(\mathrm{L}+\mathrm{TBO}+\mathrm{Au}+, \quad \mathrm{L}+\mathrm{TBO}+\mathrm{Au}-, \quad \mathrm{L}+\mathrm{TBO}-\mathrm{Au}+$, $\mathrm{L}+\mathrm{TBO}-\mathrm{Au}-, \mathrm{L}-\mathrm{TBO}+\mathrm{Au}+, \mathrm{L}-\mathrm{TBO}+\mathrm{Au}-, \mathrm{L}-\mathrm{TBO}-\mathrm{Au}+$ ) with the number of survivors from the control samples $(\mathrm{L}-\mathrm{MB}-\mathrm{Au}-)$. Likewise, the number of bacteria recovered from the $\mathrm{L}+\mathrm{TBO}+\mathrm{Au}+$ samples was compared with the viable counts obtained from the $\mathrm{L}+\mathrm{TBO}+\mathrm{Au}-$ samples. Data on surface roughness, friction coefficient, Young's modulus and singlet oxygen production were compared with the Student's $t$-test.

For all analyses, $p<0.05$ was considered statistically significant.

\section{Conclusions}

This work demonstrates that lethal photosensitisation of E. coli and MRSA can be achieved by TBO when embedded in 
polymeric materials by means of a swelling process. Greater activity was observed when the TBO was embedded in polyurethane due to higher uptake of the LAA. The presence of nanogold did not improve the antimicrobial activity in contrast with the previously reported behaviour of Methylene Blue. TBOcontaining polymers may be useful for fabricating catheters as such materials could be kept free of viable microbes by irradiation with a suitable light source.

\section{Acknowledgements}

MW, JP and IPP thank the BBSRC for supporting this work through grant BB/E012310/1, EPSRC through grant EP/ E026141/1. IPP thanks the Royal Society/Wolfson trust for a merit award.

\section{References}

1 N. Kömerik and M. Wilson, J. Appl. Microbiol., 2002, 92, 618.

2 M. Wilson, Photochem. Photobiol. Sci., 2004, 3, 412.

3 D. J. Stickler, J. C. Lear, N. S. Morris, S. M. Macleod, A. Downer,

D. H. Cadd and W. J. Feast, J. Appl. Microbiol., 2006, 100, 1028.

4 L. Ferrieres, V. Hancock and P. Klemm, FEMS Immunol. Med. Microbiol., 2007, 51, 212.

5 A. Juan-Torres and S. Harbarth, Int. J. Antimicrob. Agents, 2007, 30S, S80.

6 D. E. Norwood and A. Gilmour, J. Appl. Microbiol., 2000, 88, 512.

7 J. Kives, B. Orgaz and C. SanJose, Colloids and Surfaces B. Biointerfaces, 2006, 52, 123.
8 M. Belmares, M. Blanco, W. A. Goddard, III, R. B. Ross, G. Caldwells, S.-H. Chou, J. Pham, P. M. Olofson and C. Thomas, J. Comput. Chem., 2004, 25, 1814-1826.

9 S. Perni, C. Piccirillo, J. R. Pratten, P. Prokopovich, W. Chrzanowski, I. P. Parkin and M. Wilson, Biomaterials, 2009, 30(1), 89-93.

10 N. Stobie, B. Duffy, D. E. McCormack, J. Colreavy, M. Hidalgo, P. McHale and S. J. Hinder, Biomaterials, 2008, 29, 963.

11 T. A. Gaonkar and S. M. Modak, J. Antimicrob. Chemother., 2003, 52, 389.

12 J. Kim, B. Pitts, P. S. Stewart, A. Camper and J. Yoon, Antimicrob. Agents Chemother., 2008, 52, 1446.

13 M. R. Hamblin and T. Hasan, Photochem. Photobiol. Sci., 2004, 3, 436.

14 K. Kazmierska, M. Szwast and T. Ciach, J. Mater. Sci: Mater. Med., 2008, 19, 2301.

15 S. P. Ho, N. Nakabayashi, Y. Iwasaki, T. Boland and M. LaBerge, Biomaterials, 2003, 24, 5121.

16 F. Ahimou, M. J. Semmens, P. J. Novak and G. Haugstad, Appl. Envir. Microbiol., 2007, 73(9), 2897.

17 D. R. Absolom, F. V. Lamberti, Z. Policova, W. Zingg, C. J. Van Oss and A. W. Neumann, Appl. Environ. Microbiol., 1983, 46, 90.

18 J. Gil-Tomas, S. Tubby, N. Narband, S. Nair, M. Wilson, C. Street and I. P. Parkin, J. Mater. Chem., 2007, 17, 3739.

19 M. T. Madigan, J. M. Martinko and J. Parker, in Brock - Biology of microorganisms, Prentice Hall, 9th edn, 2000.

20 V. Decraene, J. Pratten and M. Wilson, Appl. Envir. Microbiol., 2006, 72(6), 4436.

21 C. J. van Oss, M. K. Chaudhury and R. J. Good, Chem. Rev., 1988, 88, 927.

22 R. J. Good, J. Adhesion Sci. Technol., 1992, 6, 1269.

23 F. Fischer, G. Graschew, H. J. Sinn, W. Maier-Borst, W. J. Lorenz and P. M. Schlag, Clin. Chim. Acta, 1998, 274(1), 89.

24 R. W. Horobin and J. A. Kiernan, Conn's Biological Stains, 10th edn, BIOB Scientific Publishers, Oxford, UK, 2002. 